\title{
Web Tracer: Aplicativo para monitoramento de rastreio do Mouse
}

\author{
Kennedy Edson Silva de \\ Souza \\ Universidade Federal do Pará \\ Castanhal, Brasil \\ kennedy.es.souza@gmail.com
}

\author{
Marcos César da Rocha \\ Seruffo \\ Universidade Federal do Pará \\ Belém, Brasil \\ marcos.seruffo@gmail.com
}

\author{
Harold Dias de Mello Jr. \\ Universidade do Estado do Rio \\ de Janeiro \\ Rio de Janeiro, Brasil \\ harold.dias@gmail.com
}

\begin{abstract}
RESUMO
Os modelos de plataformas de negócios geralmente exigem adaptação e agilidade contínuas para criar e oferecer novas experiências aos clientes. Para entender o comportamento do usuário em sistemas online, as pesquisas tem se aproveitado da combinação das técnicas tradicionais de análise com as mais recentes. Estudos anteriores mostraram que os dados de monitoramento do comportamento do usuário, como obtidos pelo rastreamento do mouse, podem ser usados para melhorar a experiência do usuário. Embora existam muitas soluções de rastreamento de mouse, a grande maioria é proprietária e os pacotes de código aberto não fornecem os recursos e dados necessários para dar suporte a esse trabalho. Este artigo apresenta um aplicativo para monitoramento e avaliação da experiência do usuário a partir das trajetórias e cliques do mouse. $\mathrm{O}$ aplicativo desenvolvido, intitulado Web Tracer, coleta dados e gera representações gráficas para avaliar a interação do usuário.
\end{abstract}

\section{Palavras-chave}

usuário, interface, rastreio, mouse

\section{ACM Classification Keywords}

D.2.2 Software Engineering: Design Tools and Techniques; H.5.2 Information Interfaces and Presentations (e.g., HCI): User Interfaces

\section{INTRODUÇÃO}

A transformação de modelos de negócios frequentemente faz da experiência de usuário (UX) a diferença entr o sucesso e o fracasso de um projeto. Em face disso, é preciso analisar toda a jornada do cliente nas plataformas digitais. Para isso, são necessárias ferramentas capazes de coletar dados relevantes sobre as interações entre usuários e aplicativos, especialmente com o mais comum dos periférico de entrada, o mouse.

Apesar de existirem diversos sistemas de rastreamento de mouse com uma grande variedade de recursos, tais como o MouseFlow, HotJar e CrazyEgg, estes sistemas são propri-

Permission to make digital or hard copies of all or part of this work for personal or classroom use is granted without fee provided that copies are not made or distributed for profit or commercial advantage and that copies bear this notice and the full citation on the first page. Copyrights for components of this work owned by others than the author(s) must be honored. Abstracting with credit is permitted. To copy otherwise, or republish, to post on servers or to redistribute to lists, requires prior specific permission and/or a fee. Copyright 2018 SBC.

IHC 2018, Anais Estendidos do XVII Simpósio Brasileiro sobre Fatores Humanos em Sistemas Computacionais

Outubro 22-26, 2018, Belém, Brasi

PÔSTERES VIRTUAIS E DEMOS etários. Além de imporem pagamentos recorrentes, tais ferramentas possuem método de implementação interna, isto é, exigem adaptações trabalhosas para integração de dados que não necessariamente permitiriam acesso a funcionalidades específicas dessas soluções, o que representaria severa limitação ao escopo da análise realizada no presente trabalho.

Entre as soluções não-proprietárias, destaca-se MouseTrack [1], também contando com recursos de captura e visualização de dados semelhantes aos das soluções citadas anteriormente. Todavia, os desenvolvedores alertam para possíveis incompatibilidades com sites sem certificação W3C ou que usam massivamente scripts visuais, reduzindo significativamente, portanto, o universo de aplicações testáveis.

Também cita-se Advanced web analytics tool [2] que apresenta apresenta algoritmo de filtragem e compressão de dados de interação, para diminuir a o volume de dados gerados. Tal ferramenta também não foi interessante ao presente trabalho por utilizar o mesmo método de implementação usado nas ferramentas comerciais, e por possuir como formas de representação de informação apenas a reprise das interações registradas.

Posto isso, o presente trabalho propõe uma ferramenta de captura de dados de interação de usuário gratuita e de código aberto, que permite a captura de dados sem gerar interferência nos sistemas avaliados, mesmo sem acesso ao código fonte (através de injeção de código via extensão de navegador) bem como visualização de informação através de dois modos: session replay e heatmap.

\section{METODOLOGIA}

A arquitetura da ferramenta é apresentada na Figura 1. Nessa, os dados gerados pela interação do usuário são capturados pelo módulo browser, que é dividido em três estágios, onde o motor de renderização do navegador (estágio 2) recebe os dados da página web acessada, renderiza e exibe o front-end (estágio 1) ao usuário. Ainda no estágio 2, as funções necessárias para capturar a interação do usuário com a interface são inseridas no código JavaScript do front-end. Por fim, os dados coletados no estágio 1 são enviados para o estágio 3, no qual é realizado o agrupamento dos dados e a estruturação em JSON. Posteriormente, os dados estruturados são enviados através de requisições HTTP ao servidor de armazenamento (PHP), para conversão para XML e utilização subsequente na aplicação de análise. 


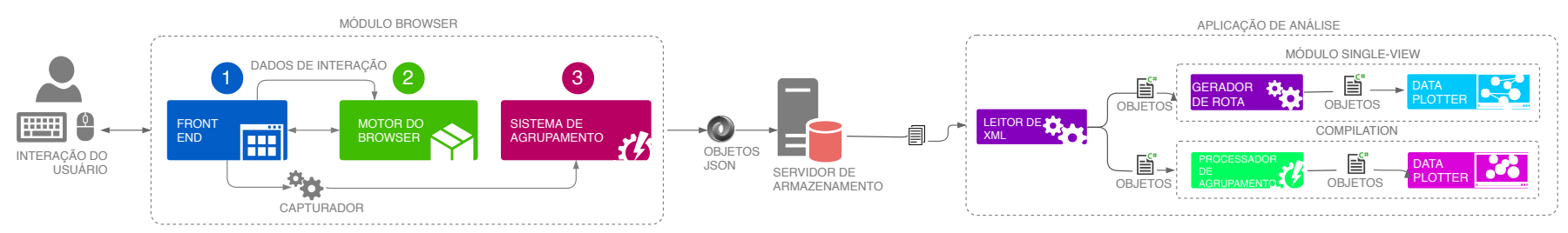

Figura 1: Visão global da arquitetura desenvolvida

Por sua vez, a aplicação de análise decodifica os dados contidos no servidor de armazenamento, para utilização em dois módulos: Single-View e Compilation. O primeiro realiza a técnica session replay, isto é, exibe quadro a quadro a sequência de passos de um usuário em sua jornada pela interface. $\mathrm{O}$ segundo módulo, por sua vez, constrói o heatmap a partir da superposição dos pontos coordenados nas telas capturadas do sistema em avaliação.

\section{RESULTADOS}

Como resultado principal deste trabalho, apresenta-se a ferramenta desenvolvida, que visa fornecer visões detalhadas sobre as interações do usuário no sistema aferido, permitindo entender como usuários interagem com sistemas Web.

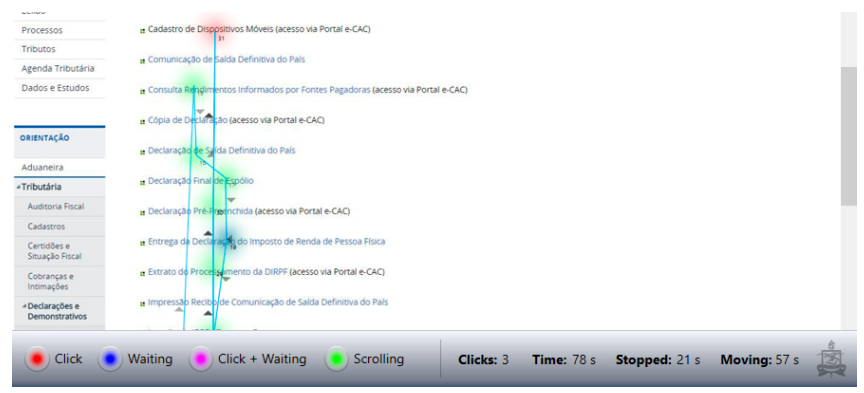

Figura 2: Módulo Single-View em execução

A Figura 2 apresenta a tela do módulo Single-View, onde observa-se o rastreamento das interações de um usuário durante a utilização do sistema. Os traços azuis conectam interações rastreadas: rolagem (pontos verdes), cliques (pontos vermelhos), esperas (pontos azuis) e cliques mesclados a esperas (pontos rosas).

Sobre cada linha de interligação, há uma seta indicando a direção da interação seguinte e sobre cada interação há uma legenda numérica, para facilitar a identificação da ordem cronológica dos eventos citados. Na barra localizada na borda inferior da referida Figura encontram-se as legendas, informando sobre a composição das representações e os dados da amostra: quantidade de cliques, tempo de ocorrência da interação atual, tempo de movimentação e inatividade do mouse.

A Figura 3 mostra o agrupamento de interações de diversos usuários através de um heatmap sobre a captura de tela do site analisado. Na borda inferior da tela mostram-se as legendas correspondentes à composição da tela, junto às informações do conjunto de amostras: quantidade total de cliques, soma dos tempos das amostras, tempo total de mouse em movimento e inativo. No lado esquerdo, juntamente com a legenda das esperas (pontos rosas) é exibida uma barra que associa a escala

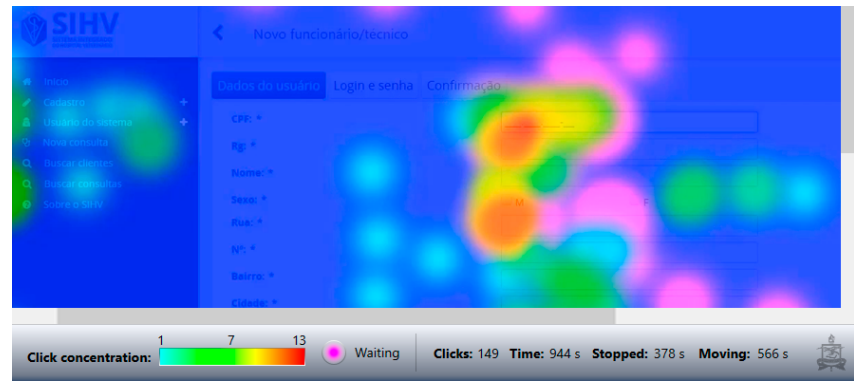

Figura 3: Módulo Compilation em execução

de cores (variando de azul a vermelho) à mínima e máxima quantidade de cliques em um mesmo local na tela.

Através do resultado apresentado, é possível identificar, por exemplo, desvios de atenção, áreas de leitura, pontos de concentração de atenção e áreas invisíveis, podendo, portanto, ser utilizado como ferramenta de verificação de efetividade de técnicas de melhoria de usabilidade, e consequentemente, de avaliação de experiência de usuário.

\section{CONCLUSÃO}

Este trabalho apresentou ferramenta de captura de dados de interação de usuário, gratuita e de código aberto, com menor risco de incompatibilidade e sem alterações no código, para entender como usuários interagem com sistemas Web. O sistema desenvolvido está disponível no repositório de código em [3].

Como trabalhos futuros, pretende-se, associado a modelos de inteligência computacional, relacionar rolagens, esperas, cliques, trajetórias e tempo utilizado, além de identificação de padrões de interação, para inferir em termos linguísticos a experiência de usuário.

\section{REFERÊNCIAS}

1. Ernesto Arroyo, Ted Selker, and Willy Wei. 2006. Usability tool for analysis of web designs using mouse tracks. In CHI'O6 extended abstracts on Human factors in computing systems. ACM, 484-489.

2. Lukáš Čegan and Petr Filip. 2017. Advanced web analytics tool for mouse tracking and real-time data processing. In Informatics, 2017 IEEE 14th International Scientific Conference on. IEEE, 431-435.

3. Kennedy Souza. 2018. KennedySouza/WebTracer. (2018). https://goo.gl/Mn4NH6 